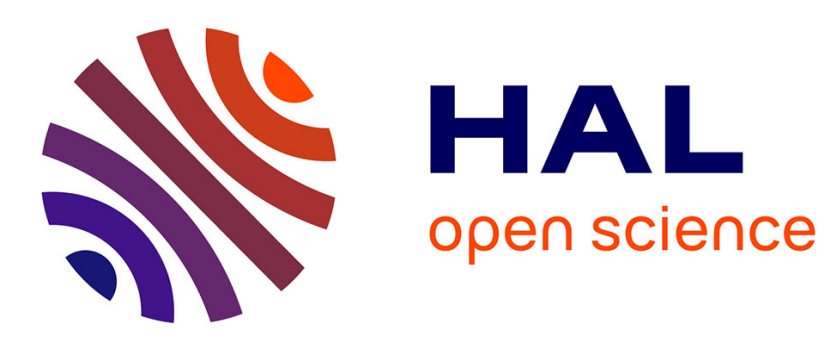

\title{
Les problématiques de diagnostic et de projection dans les PMI
}

\author{
Hervé Goy, Robert Paturel
}

\section{To cite this version:}

Hervé Goy, Robert Paturel. Les problématiques de diagnostic et de projection dans les PMI. Revue Française de Gestion, 2004, 30 (150), pp.55-70. 10.3166/rfg.150.55-70 . halshs-00675774

\section{HAL Id: halshs-00675774 \\ https://shs.hal.science/halshs-00675774}

Submitted on 6 Mar 2012

HAL is a multi-disciplinary open access archive for the deposit and dissemination of scientific research documents, whether they are published or not. The documents may come from teaching and research institutions in France or abroad, or from public or private research centers.
L'archive ouverte pluridisciplinaire HAL, est destinée au dépôt et à la diffusion de documents scientifiques de niveau recherche, publiés ou non, émanant des établissements d'enseignement et de recherche français ou étrangers, des laboratoires publics ou privés. 


\section{Les problématiques de diagnostic et de projection dans les PMI}

Quelle est l'attitude actuelle des PMI françaises à l'égard des pratiques de planification? Cette question est abordée en revenant sur la littérature se rapportant à la planification, afin d'élaborer un cadre d'analyse pertinent susceptible de faciliter I'observation des pratiques dans les PMI. À partir d'une méthodologie quantitative, les résultats issus de l'analyse de 82 situations permettent de mieux comprendre la réalité de la planification et, notamment, de lister les variables de contingence mises en avant. L'article débouche sur un essai de classification typologique des PMI en fonction de leurs attitudes et de leurs pratiques face à la planification.
T e questionnement de la pertinence des pratiques de planification dans le contexte particulier des PME n'est certes pas nouveau, mais il semble se poser de façon récurrente: on peut penser que la quasi-intégration, voire l'intégration des PME dans de grands ensembles, ou bien encore leur contrôle financier et logistique n'y sont pas étrangers, ce à quoi il faudrait également ajouter les vagues de normalisation de type ISO. Bien entendu, le débat n'est ni neuf (dans la RFG tout particulièrement, avec l'article de Calori, Very et Arregle en 1997), ni clos (Goy, 2001).

À travers l'étude de la planification en PME, deux constats doivent d'emblée être dressés: tout d'abord, nous ne pouvons que souscrire au point de vue de Marchesnay (1997) sur l'extrême diversité des configurations actuelles « des » PME. Quand bien même on peut admettre une définition idéal type de «la »PME, le rôle du dirigeant reste essentiel sur la question posée dans cet article. Ensuite, il convient d'admettre l'existence, dans les PME, de divers niveaux de planification, depuis les procédures très formalisées, jusqu'à de 
simples processus « visionnaires », en passant par les business plan et autres «projets d'entreprise ». Comme l'a notamment montré Torrès (1997), bien des PME sont gérées de façon managériale, et sont amenées à manipuler des outils de planification (par exemple, un sous-traitant de spécialité travaillant avec un groupe industriel).

Dès lors, notre problématique consiste à s'interroger sur l'état actuel des pratiques des PME, sur un échantillon d'entreprises industrielles, en matière de planification. Ce questionnement nous conduira à un essai de classification typologique des PMI en fonction de leurs attitudes et de leurs pratiques face à la planification. Pour ce faire, nous aborderons dans un premier temps le traitement, par la littérature en stratégie, des pratiques de planification dans les PME. Nous soulèverons, à ce propos, les divergences qui existent quant à l'évaluation du comportement planificateur de ces firmes, ce qui nous conduira à formuler un cadre d'analyse conceptuel cohérent, susceptible de permettre l'observation empirique des pratiques de planification dans les PME. Puis, nous serons amenés à présenter, dans un second temps, les grandes lignes de la méthodologie qui nous a permis d'aboutir à une typologie des pratiques de planification dans les PMI de notre étude, qui viendront conclure notre propos.

\section{I. - PLANIFICATION ET PME, DEUX NOTIONS À (RE)CONCILIER}

Plusieurs études ont été conduites afin de tendre vers une meilleure appréhension des pratiques de planification des PME. Pour autant, le consensus semble difficile, tant les approches retenues divergent, aussi bien en termes de méthode que de définitions données à la planification. Nous tenterons donc de saisir ces disparités. Cela nous conduira, par la suite, à proposer une grille de lecture conceptuelle de la planification.

\section{Des recherches inductives stériles}

Des conceptions pour le moins variées de la planification

L'existence de « la planification »

De nombreux travaux ont cherché à démontrer le caractère plus ou moins répandu de la planification en PME. C'est ainsi qu'un résultat-clé de la recherche de Robinson et Pearce (1984), rappelé par Bracker et Pearson (1986), est que la majorité des PME ne planifie pas. De même pour Bamberger (1980), les méthodes de planification ne sont guère utilisées en PME (seulement $24 \%$ des entreprises interrogées), et correspondent plutôt aux caractéristiques, besoins et ressources de la direction des grandes entreprises. Shaw, Shuman et Sussman (1986) estiment que seulement $30 \%$ des PME ont recours à la planification stratégique, par processus organisé et structuré de manière générale. Calori, Véry et Arrègle (1997) parviennent à un résultat comparable auprès de PMI françaises: $30 \%$ d'entre elles pratiquent la planification stratégique (selon des degrés d'anticipation et de formalisation forts).

En revanche, d'autres travaux ont conclu à des résultats plus nuancés. Par exemple, Waalewijn et Segaar (1993) estiment que $80 \%$ des PME ne font pas de plans à long terme et, dans le même temps, constatent que la plupart font de la planification financière. De la même façon, Sexton et Dahle (1976) soulignent que la planification à long 
terme en PME est plus répandue que ce que l'on croit, bien que le processus ne soit pas aussi sophistiqué que dans les grandes entreprises. Sexton et Van Auken (1985) confirment pour leur part l'idée selon laquelle la planification en PME est sporadique. Dans le même ordre d'idées, pour Shrader, Mulford et Blackburn (1989), un tiers des PME ont un plan stratégique, et toutes sont engagées dans une forme de plan opérationnel. Enfin, Robinson, Logan et Salem (1986), dans une investigation sur la relation entre la planification opérationnelle et la planification stratégique des PME, ont montré que $85 \%$ des entreprises étudiées (des détaillants) ne pratiquent pas systématiquement la planification stratégique.

Les causes des différentes formes de planification

Selon Shuman (1975), la principale justification de la nature informelle et non structurée des activités de planification est la faible taille de l'entreprise (les avantages procurés par un processus plus structuré ne dépasseraient pas les sacrifices et efforts nécessaires à sa mise en œuvre). De même, pour Shrader, Mulford et Blackburn (1989), comme le dirigeant est le plus souvent le seul impliqué dans le processus de planification stratégique, celle-ci s'apparente à du «tout ou rien » dans les petites et moyennes entreprises (pas de plans écrits ou planification sophistiquée). Enfin, Shuman, Shaw et Sussman (1985) estiment qu'avec la croissance, d'une part, le processus de planification utilisé devient plus formel, structuré et participatif, bien que ce processus reste éloigné de ceux décrits dans la littérature; et d'autre part, la planification stratégique tend à être orientée à court terme, opérationnelle et mise à jour régulièrement.
L'ensemble des travaux consultés, bien que riches, laisse cependant le sentiment que d'une étude à l'autre, on ne traite pas forcément des mêmes concepts. Examinons dans quelle mesure les définitions données à la planification rendent envisageable la comparaison de ces différentes recherches.

\section{Des mesures de la planification}

\section{trop divergentes}

Plusieurs chercheurs ont tenté de délimiter «le phénomène planification » dans les PME. Le tableau synoptique suivant synthétise les principaux axes de recherche en la matière.

Hormis les travaux de Robinson et al. (1984), qui caractérisent l'intensité de la planification par le nombre d'heures investies ou bien encore le nombre de consultants impliqués, la majorité de ces études sur la planification en PME se focalise surtout sur l'existence ou non d'un plan écrit, réduisant de fait à l'écriture le caractère plus ou moins formel de la planification. À cela, on pourrait opposer le point de vue de Tanguy (1992), pour qui « même en l'absence de la formalisation d'un plan écrit, voire d'un service attaché à cette fonction, la planification se manifeste sous des formes multiples: c'est le chiffrage sur un coin de table des projets du dirigeant qui va servir à orienter ses troupes, ou encore la discussion qui va conduire aux arbitrages budgétaires ».

Cette rapide revue de quelques-unes des approches retenues pour mesurer le degré de planification en PME atteste de divergences dans les méthodes utilisées. Ce manque de cohésion s'explique principalement, selon nous, par le fait que les différents auteurs considèrent la planification comme un outil de gestion, alors qu'elle 


\begin{tabular}{|c|c|}
\hline \multicolumn{2}{|c|}{$\begin{array}{c}\text { Tableau } 1 \\
\text { FACTEURS D'IDENTIFICATION DE LA PLANIFICATION EN PME }\end{array}$} \\
\hline Auteurs & \\
\hline $\begin{array}{l}\text { Montebello, Saias et } \\
\text { Greffeuille (1974) }\end{array}$ & $\begin{array}{l}\text { - Importance accordée à l'étude de l'environnement et au } \\
\text { diagnostic interne. } \\
\text { - Définition d'objectifs stratégiques à long terme. } \\
\text { - Choix de manœuvres pour atteindre ces objectifs. } \\
\text { - Formalisation de l'ensemble par écrit. }\end{array}$ \\
\hline Sexton et Van Auken (1985) & $\begin{array}{l}\text { - Absence de prévision à un an des ventes, de la rentabilité et de } \\
\text { plans d'implémentation (niveau 0). } \\
\text { - Prévision des ventes seulement (niveau 1). } \\
\text { - Niveau } 1 \text { + prévision des ventes du secteur (niveau 2). } \\
\text { - Niveau } 2 \text { + anticipation du profit de l'entreprise (niveau 3). } \\
\text { - Niveau } 3 \text { + plans de mise en œuvre (niveau 4). }\end{array}$ \\
\hline Robinson et al. (1984) & $\begin{array}{l}\text { - Aborder les problèmes de la stratégie selon les niveaux } \\
\text { d'activité. } \\
\text { - Inclure dans l'analyse et la prise de décision deux fonctions ou } \\
\text { plus de l'entreprise. } \\
\text { - Entretenir dix heures ou plus de contacts entre clients et } \\
\text { consultants. } \\
\text { - Inclure au moins trois périodes de contact distinctes avec les } \\
\text { consultants. }\end{array}$ \\
\hline $\begin{array}{l}\text { Shrader, Mulford et } \\
\text { Blackburn (1989) }\end{array}$ & $\begin{array}{l}\text { - Pas de plan stratégique écrit pour l'année à venir. } \\
\text { - Plan stratégique écrit (objectifs, allocation des ressources, } \\
\text { stratégies à long terme). } \\
\text { - Plan stratégique écrit, accompagné d'une analyse des facteurs } \\
\text { environnementaux et de mesures correctives des écarts } \\
\text { (planification sophistiquée). }\end{array}$ \\
\hline
\end{tabular}

doit être envisagée comme un dispositif de gestion, au même titre que la veille ou la gestion de projet. Dès lors, la planification s'apparente à une activité qui agit sur un objet, et non à un objet en soi. Ce postulat nous amène donc, dans une seconde section, à formuler un cadre d'analyse théorique de la planification, sur lequel nous entendons par la suite appuyer notre étude empirique.

\section{La nécessité de recourir à une approche conceptuelle nouvelle de la planification}

L'approche managériale, procédurale, systématique, voire technique de la planification n'est pas nécessairement adaptée aux pratiques et objectifs des PME, ce qui implique de tenir compte des aspects cognitifs (en termes de logiques d'action), des démarches processuelles (heuristique), du caractère 
systémique, etc. Dès lors, le problème auquel on se retrouve rapidement confronté est celui de l'identification de la planification dans son contexte: quelles dimensions permettent d'affirmer que telle entreprise planifie, et telle autre non? En d'autres termes, il s'agit de repérer les phases récurrentes des processus de planification. Nous chercherons donc à synthétiser les principales étapes de toute démarche de planification, ce qui nous amènera à proposer un modèle conceptuel sur lequel nous fonder.

\section{Pas de planification sans le triptyque} «finalisation - programmation - action»

Trois éléments nous paraissent constituer le cœur de tout processus de planification: la fixation des buts et objectifs, la réalisation de programmes ainsi que la phase de mise en œuvre (ou action).

La fixation des buts et objectifs organisationnels

Si cette étape est évoquée dans la plupart des modèles de planification traditionnels, il ne semble pas exister de consensus sur la hiérarchie entre buts et objectifs. Tabatoni et Jarniou (1975) définissent les objectifs comme « certaines formes d'orientation volontaire de l'action humaine exprimées en termes plus opérationnels que d'autres ». De même, Ozbekhan (1969) parle pour sa part de buts, «issues générales, lointaines, idéales », caractérisés par leur universalité, leur relativité ainsi que par leur éthique. Au contraire, pour Vancil (1977) « un objectif est une aspiration à modeler dans l'avenir », et « un but est un accomplissement qui doit être atteint à une certaine date future ». Les fins sont définies par Ackoff (1973) comme la « spécification des objectifs et des buts ». Toutefois, la tendance générale consiste à assimiler le but à une intention, à une aspiration et l'objectif à la concrétisation d'un but donné par le biais d'une cible, d'un horizon temporel et d'un critère de mesure dont un niveau doit être atteint.

\section{La programmation}

Certains parlent de programmes (Steiner, 1979; Mintzberg, 1994), d'autres de réalisations (Martinet, 1983). Le terme de programmation définit l'étape qui s'intercale entre la finalisation et l'action. Pour Desreumaux (1993), cette phase traduit la transition entre planification stratégique et planification opérationnelle. Plus concrètement, il s'agit de savoir si, entre la définition de la direction à suivre et les actions envisagées pour réaliser ces buts et objectifs, des séquences d'opérations sont définies.

\section{L'action}

Cette dimension présuppose que l'effort de planification ne reste pas «lettre morte », mais soit, au contraire, concrétisé par un certain nombre d'actions. Pour Mintzberg (1994), « planifier, c'est contrôler le futur; pas uniquement $\mathrm{y}$ penser, mais agir dessus. »

À partir du moment où ces trois étapes sont isolées dans une organisation, selon des degrés variables, nous estimons qu'elle planifie effectivement. Mais d'autres fonctions viennent se greffer autour de ce noyau dur.

\section{Des dimensions périphériques importantes}

Deux autres sous-systèmes nous semblent inhérents à tout processus de planification: le contrôle ex-post et l'analyse ex-ante.

Le contrôle ex-post

Cette fonction de la planification n'est pas toujours apparente. Ainsi, Mintzberg (1994) semble conclure son « diagramme 
des quatre hiérarchies » par la dimension « actions ». Pour Thiétart (1990), en revanche, la « comparaison des objectifs aux résultats » semble occuper une place importante dans le processus, comme c'est également le cas pour Steiner (1979) avec « l'étude et évaluation des plans ». Selon Drucker (1959), le contrôle est clairement le point de départ d'un nouveau cycle de planification, conduisant à la prise de nouvelles décisions. Pour Ackoff (1973), le contrôle est « la conception d'une méthode d'anticipation et de détection des erreurs ou des échecs commis dans le plan, et d'une méthode de prévention et de correction permanente de ceux-ci ». Nous retiendrons pour notre part l'idée de contrôle en aval de la dimension d'action, qui peut revêtir des formes et une importance variables en fonction des situations organisationnelles.

\section{L'analyse ex-ante}

D'après la classification de Mintzberg (1994), le modèle de base de la planification est celui de «l'école de la conception ». Ce modèle se caractérise notamment par sa double dimension d'analyse (interne et externe), bien connue sous le nom d'analyse « SWOT ». Le schéma proposé par Martinet (1983) reprend ce principe, qui conduit au « diagnostic de la position stratégique ». La représentation de Ansoff (1965) inclut de la même manière cette analyse ( « évaluation interne » et « évaluation externe »), tout comme celle de Steiner (1979), avec l'évaluation des opportunités externes et des problèmes, et des forces et faiblesses de l'entreprise en interne. Pour notre part, nous retiendrons le terme de diagnostic pour caractériser la dimension d'analyse de l'environnement interne et externe de l'entreprise, en amont de la phase de finalisation. Notre choix est représenté dans la figure 1.

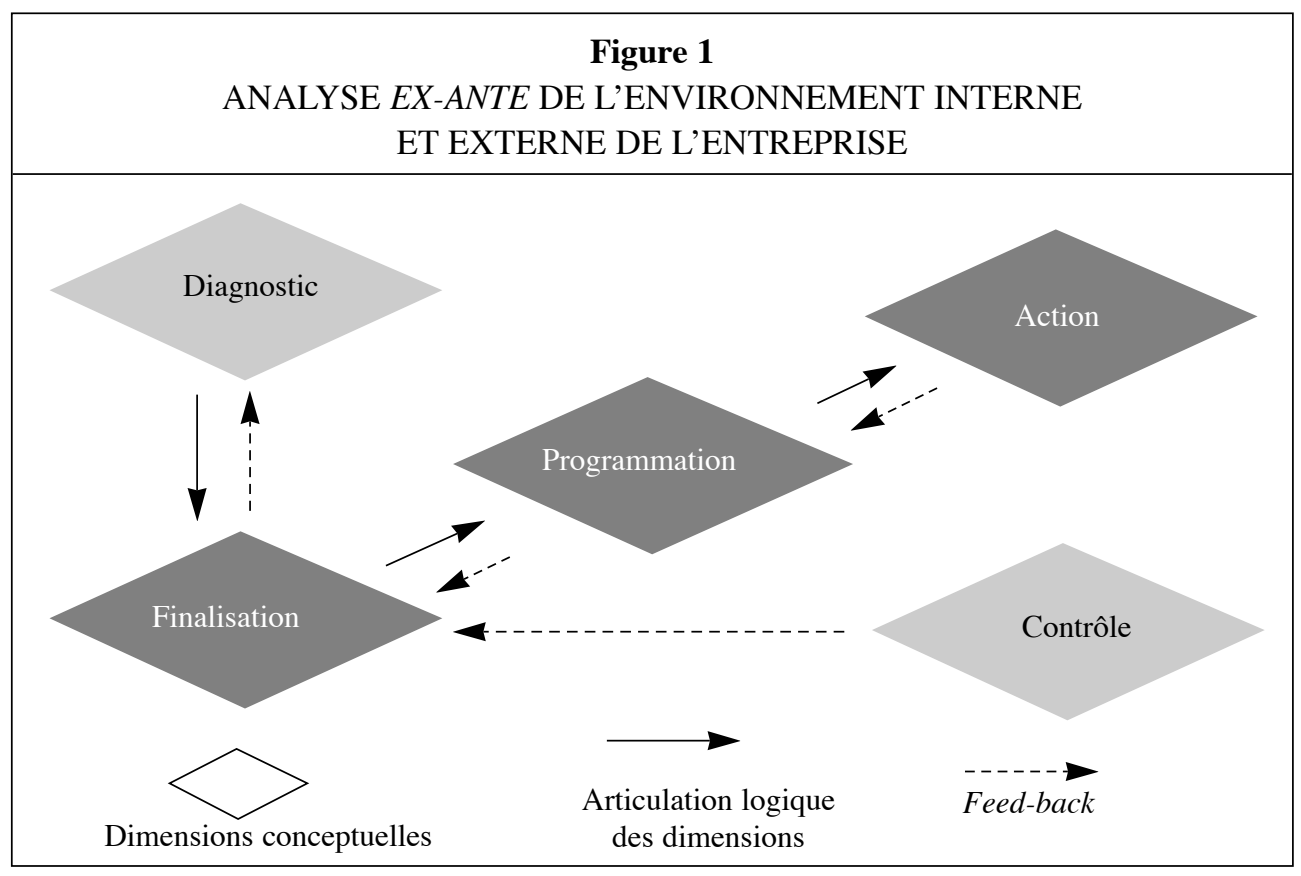


Bien entendu, et même si nous n'insistons pas sur ce point, nous avons conscience du rôle et de l'influence que l'entrepreneur peut jouer dans la mise en œuvre concrète de ce schéma (Paturel, 1999).

\section{Notre proposition d'identification} des pratiques de planification

Nous avons donc choisi de caractériser toute démarche de planification selon l'articulation de cinq dimensions : trois d'entre elles constituent le cœur du processus, en l'absence desquelles, selon nous, on ne peut parler de planification, et les deux autres restent d'une importance relative, selon les situations organisationnelles. En d'autres termes, nous retiendrons qu'une organisation planifie dès lors que l'on peut identifier l'existence (plus ou moins impor- tante selon le degré de réalisation) de soussystèmes de finalisation (l'établissement de buts et d'objectifs), de programmation (la définition de séquences d'opérations à implémenter) et d'action (le « passage à l'acte » ou action sur « l'objet planifié »). Les phases de diagnostic et de contrôle, dont on imagine mal qu'elles soient totalement absentes des préoccupations des dirigeants, peuvent cependant ne pas être clairement intégrées et articulées dans le processus: c'est pourquoi, leur faible représentation éventuelle ne remet pas en cause, selon nous, l'existence de pratiques de planification. Le schéma de la figure 2 résume notre positionnement.

Ce choix implique des conséquences en termes de positionnement théorique qu'il nous semble important de souligner. Tout

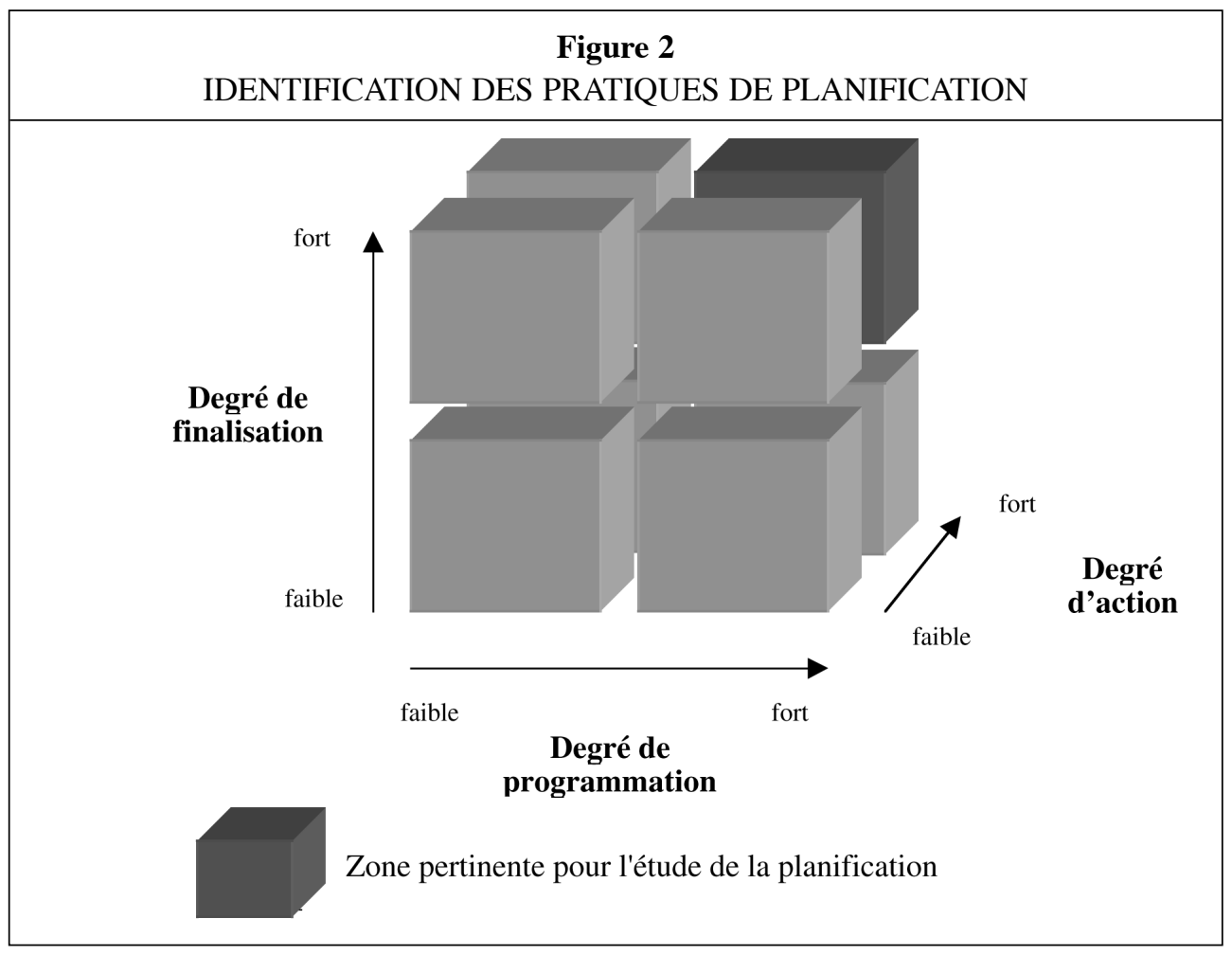


d'abord, nous nous situons en rupture avec les approches fondées sur l'identification de la planification par sa forme. En d'autres termes, la présence de documents écrits ou de procédures formalisées sont autant de critères insuffisants, selon nous, pour caractériser le comportement planificateur d'une entreprise, et notamment d'une PME. Ensuite, nous préférons utiliser le terme de planification à celui de planification stratégique, trop lourd de sens, notamment suite à la connotation très formalisée que Mintzberg (1994) a contribué à lui donner. De fait, nous n'avons aucun a priori sur l'objet auquel la planification a trait: il peut aussi bien s'agir de la stratégie de l'entreprise que du fonctionnement de ses affaires courantes, de l'ensemble des grandes fonctions de l'organisation que de l'une d'entre elles. En ce sens, nous nous inscrivons pleinement dans l'approche de Ozbekhan (1969), pour qui la planification est une activité indépendante de l'objet auquel elle s'attache. Sur cette base conceptuelle, nous pouvons dès lors envisager une étude de terrain. Les modalités de cette démarche, ainsi que ses principaux résultats, sont maintenant détaillées.

\section{II. - L'IDENTIFICATION DE QUATRE PROFILS DE PLANIFICATEURS EN PMI}

Les trois conditions nécessaires, selon notre approche, pour identifier un comportement planificateur sont-elles réunies dans le cadre des PMI? Si oui, quelles sont les attitudes vis-à-vis de ces pratiques? Voici les questionnements qu'il s'agit d'éclairer, selon le protocole exposé à présent.

\section{Les choix de notre protocole de recherche}

Afin de répondre à notre problématique d'identification des attitudes et des pratiques actuelles des PMI en matière de planification, la logique quantitative semble s'imposer. En effet, comment aboutir à un essai de classification typologique, sans chercher à en caractériser les principaux éléments auprès d'un nombre suffisant d'entreprises? En d'autres termes, la réponse à notre interrogation, passe selon, nous par la réalisation d'une photographie suffisamment large des comportements planificateurs des PMI, dont le résultat puisse faire l'objet d'un traitement statistique.

Nous avons donc entrepris d'élaborer un questionnaire permettant d'évaluer le degré de pratiques de planification par les PMI. Pour ce faire, plusieurs choix ont du être effectués, sur la base des arguments suivants. Nous avons tout d'abord choisi de nous intéresser aux petites et moyennes entreprises industrielles (ce qui, en soi, représente déjà un nombre considérable d'activités). Ce choix peut s'expliquer par l'analyse des terrains de recherche mobilisés dans les travaux antérieurs sur la planification des PME. En effet, la majorité des études sur les entreprises de services ne nous paraissent pas reproductibles en France: c'est le cas de Robinson et Pearce (1983) ou Kargar (1996) avec les banques américaines, et de Robinson, Logan et Salem (1986) ou Shrader, Mulford et Blackburn (1989) avec les commerces de détail alimentaires.

Nous avons ensuite retenu des entreprises de Rhône-Alpes, qui occupe une place de choix dans le classement économique des régions françaises. De même, on peut raisonnablement penser que ces PMI sont 
assez représentatives de l'ensemble des entreprises du territoire. Enfin, on peut dénombrer dans cette zone une proportion importante de PMI (près de $40 \%$ selon le critère de l'effectif retenu ci-après).

Enfin, nous avons décidé de considérer qu'une PMI, d'un point de vue quantitatif, emploie entre 10 (en deçà, Julien et Marchesnay (1988) considèrent que les TPE ont un comportement suffisamment particulier pour constituer une catégorie à part) et 250 salariés, la limite supérieure retenue par la norme européenne (Marchesnay, 1997).

\section{Présentation de l'échantillon de PMI obtenu}

Sur la base de ces choix, nous avons donc administré 500 questionnaires par voie postale auprès de responsables (P-DG, DG, DAF, DRH, DC, etc.) de PMI, tirées au sort de la population de départ (4024 entreprises au total issues de la base de données économiques DIANE). Après relance, nous avons comptabilisé 101 retours (soit un taux de réponses de $20,2 \%$ ), dont 82 exploitables, caractérisés dans le tableau 2 .

\begin{tabular}{|c|c|}
\hline \multicolumn{2}{|c|}{ Tableau 2 } \\
\hline \multicolumn{2}{|c|}{ ÉCHANTILLON DE PMI } \\
\hline Taille de l'échantillon & 82 entreprises \\
\hline - dont entre 10 et 49 salariés & 41 entreprises (50\%) \\
\hline - dont entre 50 et 99 salariés & 21 entreprises $(25,6 \%)$ \\
\hline- dont entre 100 et 250 salariés & 20 entreprises $(24,4 \%)$ \\
\hline
\end{tabular}

La mise en œuvre de nos choix conceptuels nous a conduit à isoler, parmi les 82 PMI répondantes, la part de «planificateurs » (pour davantage de précisions, nous renvoyons le lecteur aux travaux de Goy, 2000):

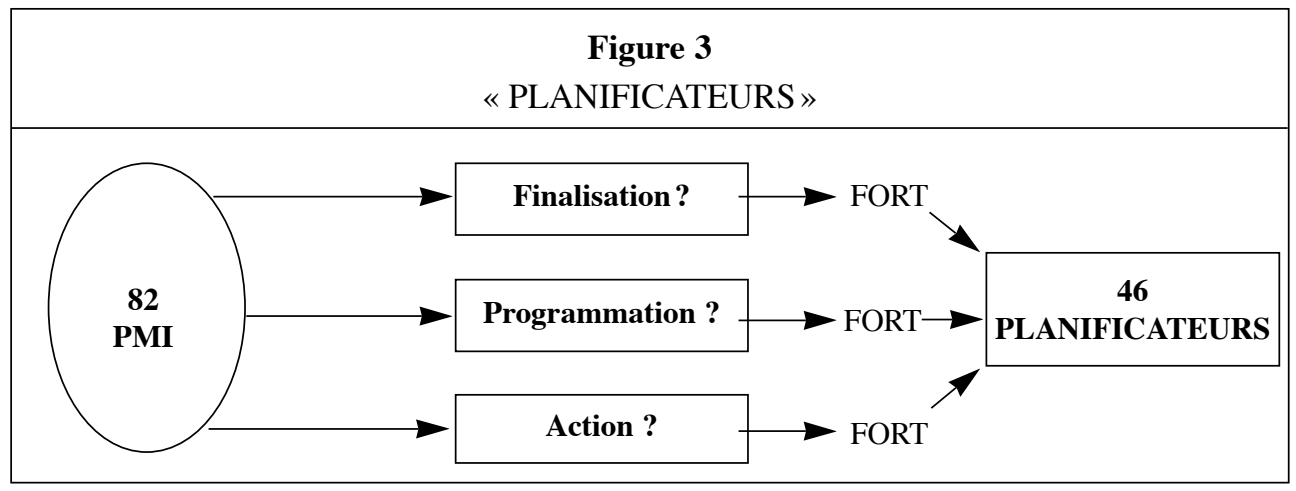


Il convient de préciser que d'un pur point de vue statistique, cet échantillon ne peut être considéré comme représentatif: outre le nombre relativement faible de PMI planificatrices, on peut raisonnablement subodorer, en effet, que dans les non-réponses, le nombre de «non-planificateurs » est important.

Pour autant, nous avons pu distinguer, sur la base des affirmations des répondants, l'existence de sous-systèmes de finalisation (définition de buts et d'objectifs), de programmes de mise en œuvre et de réalisation effective des objectifs auprès de $56 \%$ des PMI interrogées. Bien entendu, à ce stade de l'analyse, ces pratiques de planification peuvent concerner tout ou partie des fonctions de l'entreprise, tout ou partie des décisions prises aux différents niveaux de responsabilité, etc. Pour autant, du fait de notre positionnement conceptuel, ce premier résultat tranche avec certains travaux évoqués précédemment, qui minimisent l'existence de la planification dans ce type d'entreprise. Nous développerons quelques-uns des traits les plus significatifs des PMI planificatrices.

\section{La taille}

En termes d'effectifs, les PMI qui planifient ont une dimension supérieure aux autres. Ce résultat est confirmé par l'analyse du chiffre d'affaires, qui tend vers la même conclusion. Toutefois, la fourchette est large, puisque plus de la moitié des entreprises (tous modes de gestion confondus) emploie moins de 50 salariés ou réalise moins de 8 millions d'euros de chiffre d'affaires. De la même façon, les PMI de plus de 100 salariés ou 15 millions d'euros de chiffre d'affaires représentent un peu moins du quart de notre échantillon.

\section{La détention du capital}

Près d'une PMI sur deux qui planifie est rattachée à un groupe, alors que les PMI qui ont d'autres pratiques de gestion sont davantage indépendantes. Ce point renforce le principe d'une planification managériale, que nous allons approfondir plus avant.

\section{Les aspirations du dirigeant}

L'aspiration des PMI vis-à-vis de la conservation de leur autonomie dans la prise de décision semble indépendante $\mathrm{du}$ fait qu'elles planifient ou non. En revanche, on constate un très net penchant des PMI qui planifient à rechercher la croissance de leur entreprise (lien statistiquement significatif). De même, les non planificateurs n'étant pour la majorité pas rattachés à un groupe, il semble assez logique qu'ils préfèrent davantage que les planificateurs conserver cette situation d'indépendance patrimoniale. Enfin, la pérennité de l'entreprise semble être primordiale pour les dirigeants de PMI de manière générale, et plus particulièrement pour les firmes n'ayant pas de pratiques de planification.

Les «vraies » et «fausses » PMI

Du point de vue de l'indépendance de gestion, près d'une PMI sur deux qui planifie (environ $44 \%$ ) est rattachée à un groupe, alors que les PMI qui ont d'autres pratiques de gestion sont davantage indépendantes (à plus de $72 \%$ ). Ce résultat présuppose l'existence de pratiques de planification managériale dans la plupart des entreprises concernées. Sur la question de l'autonomie des entreprises interrogées dans leur management, les PMI qui planifient semblent relativement moins détenir la possibilité de déterminer leur stratégie à 


\begin{tabular}{|c|c|c|c|c|}
\hline \multicolumn{5}{|c|}{$\begin{array}{c}\text { Tableau } 3 \\
\text { CHOIX DE LA STRATÉGIE POUR 46 PMI }\end{array}$} \\
\hline & & \multicolumn{2}{|c|}{ Autonomie dans le choix de la stratégie } & \\
\hline & & Faible & Forte & TOTAL \\
\hline \multirow{2}{*}{$\begin{array}{l}\text { Autonomie dans } \\
\text { le choix des } \\
\text { procédures }\end{array}$} & Faible & $\begin{array}{c}\text { Planification } \\
\text { managériale } \\
\text { globale (3 PMI) }\end{array}$ & $\begin{array}{c}\text { Planification } \\
\text { managériale partielle } \\
\text { (2 PMI) }\end{array}$ & 5 entreprises \\
\hline & Forte & $\begin{array}{c}\text { Planification } \\
\text { managériale } \\
\text { partielle (6 PMI) }\end{array}$ & $\begin{array}{c}\text { Planification } \\
\text { entrepreneuriale } \\
\text { (35 PMI) }\end{array}$ & 41 entreprises \\
\hline \multicolumn{2}{|c|}{ TOTAL } & 9 entreprises & 37 entreprises & 46 PMI \\
\hline
\end{tabular}

venir que les autres (près de $20 \%$ d'entre elles déclarent ne pas définir la stratégie de leur entreprise). En revanche, que les entreprises planifient ou non, elles semblent avoir la même latitude dans le choix des méthodes de réalisation de la stratégie préalablement définie. Dès lors, il semblerait que l'on puisse parler de planification entrepreneuriale lorsque les PMI sont maîtresses à la fois de la définition et de la mise en œuvre de leur stratégie, et de planification managériale quand les entreprises n'ont plus de latitude en matière de détermination stratégique. Le tableau 3 synthétise ces différentes possibilités, auprès des 46 PMI planificatrices de notre étude.

Aussi instructive soit-elle, l'étude de ces variables contingentes ne nous permet cependant pas d'appréhender les probables variations dans l'attitude des 46 planificateurs. En revanche, une analyse typologique permet d'approfondir ce point, que nous allons présenter pour conclure.

\section{Les comportements des PMI planificatrices, entre analyse et projection}

La mise en évidence des attitudes des dirigeants de PMI face à la planification passe par la réalisation d'une analyse typologique Il existe essentiellement deux méthodes de classification, dont la combinaison permet d'approcher un résultat satisfaisant.

- La méthode agrégative hiérarchique (ou ascendante) aboutit à un arbre de classification (ou dendrogramme) utilisé pour repérer le nombre de groupes d'entreprises ayant un comportement semblable (figure 4).

- La méthode agrégative non hiérarchique permet de tester, parmi les solutions à trois ou quatre groupes mises en avant par la classification hiérarchique, celle qui convient le mieux. Du point de vue de la validité statistique de la solution retenue au moyen de l'analyse de variance, il convient de conserver quatre groupes de planificateurs, tous les tests étant significatifs à un seuil de $5 \%$. C'est pourquoi, compte tenu 


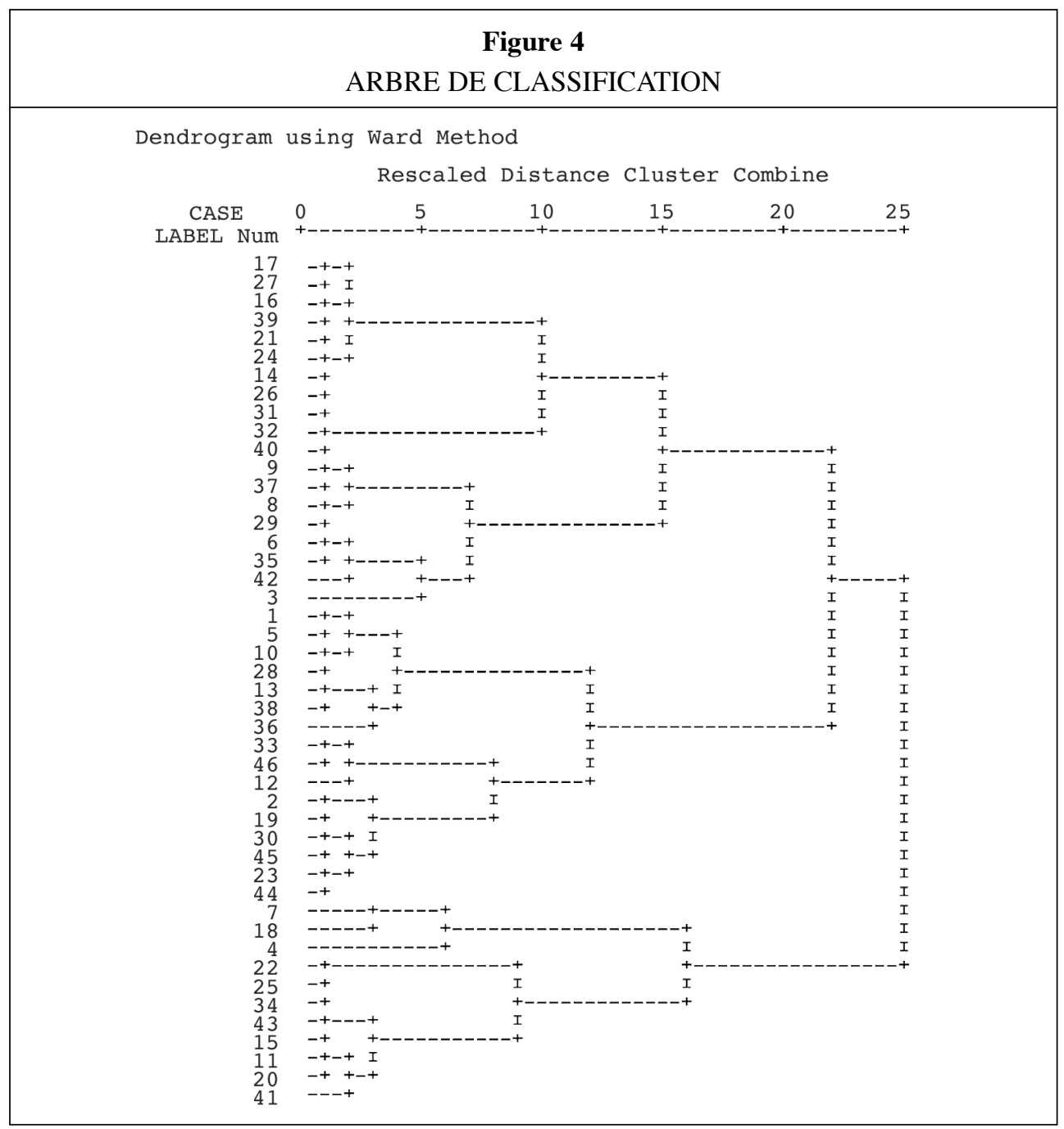

de l'information procurée par ces analyses, nous retenons la solution à quatre groupes, correspondant à quatre types d'attitudes des 46 PMI face à la planification, et qu'il s'agit d'interpréter à présent. Pour ce faire, à la lecture des barycentres les plus significatifs des centres finaux de classes, en valeur absolue arrondie et pour chaque classe, on aboutit à l'interprétation suivante :
- Le premier groupe rassemble des PMI qui se caractérisent à la fois par un faible recours à des outils de diagnostic stratégique (de type segmentation ou matrices, par exemple), une faible projection dans l'avenir en termes de fixation de buts et d'objectifs, mais en revanche, par une forte tendance à mettre l'accent sur les différentes étapes de l'implémentation (mise en 
œuvre des décisions, contrôle de l'état d'avancement des réalisations, procédures correctrices le cas échéant). Ainsi, la devise de ce groupe pourrait se résumer par «l'action avant tout! » Nous le nommerons donc le groupe des planificateurs « acteurs ».

- Le deuxième groupe rassemble des PMI dont la tendance semble consister à rejeter ou, en tout cas, à ne pas rechercher, le court terme, dans le sens de la détermination d'objectifs de gestion à réaliser dans un horizon rapproché (six mois au maximum). La contrepartie de cette aspiration réside dans la réalisation d'un diagnostic interne de la situation assez significatif, sur la base d'instruments d'analyse relativement classiques (dans tous les cas, au moins une segmentation des différentes activités de l'entreprise est définie). Le mot d'ordre de ce groupe pourrait être «pas de préci- pitation! 》 Aussi appellerons-nous ces entreprises les planificateurs « analystes ». - Le troisième groupe se caractérise essentiellement par l'attention portée à l'environnement externe de l'entreprise, qu'il soit spécifique (la filière, les concurrents) ou global (économie, géopolitique, etc.). L'accent est beaucoup moins mis sur les dimensions de finalisation et de mise en œuvre (surtout dans le sens de la préparation, de la programmation des étapes). L'expression « voyons ce que font les autres » pourrait résumer la philosophie de ce groupe, que nous nommerons les planificateurs « observateurs ».

- Le dernier groupe, enfin, est composé d'entreprises dont la préoccupation n'est a priori pas de s'intéresser à ce qui se passe autour d'elles, mais plutôt de chercher à se projeter dans un avenir plus ou moins lointain, sur la base de l'élaboration d'une cer-

Figure 5

QUATRE TYPES DE PLANIFICATEURS

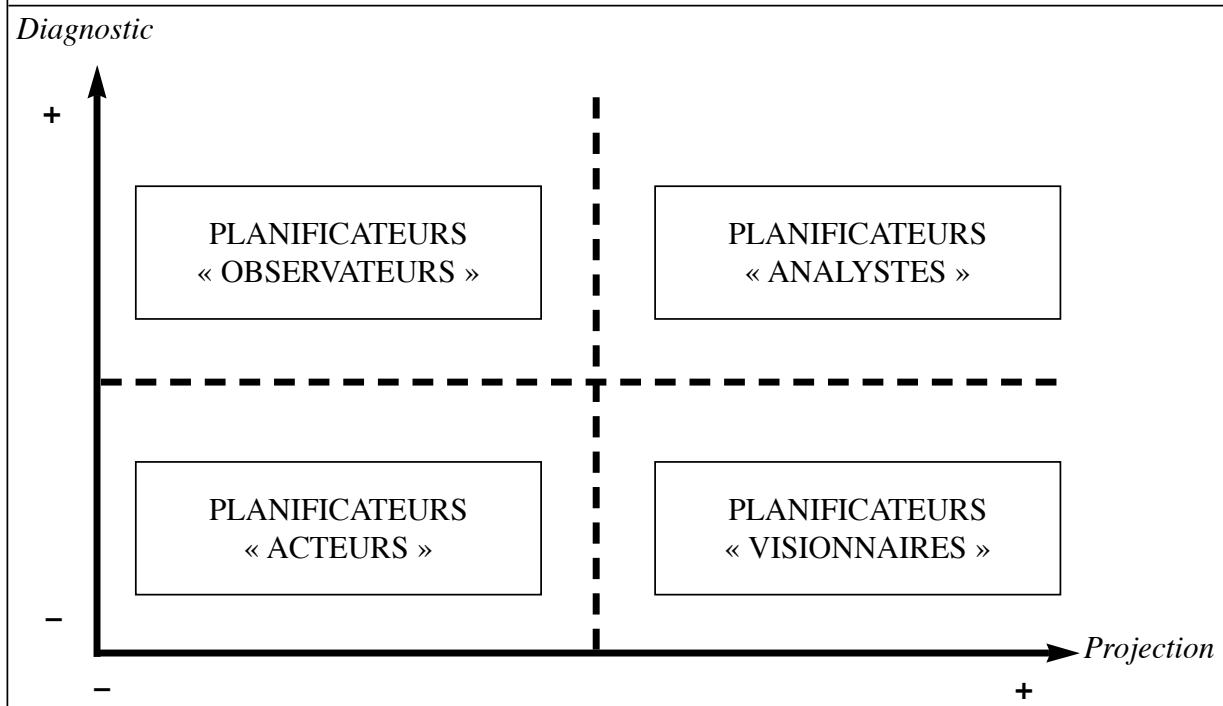


taine vision du développement de l'organisation. «Voici où nous devrons être dans $x$ années » pourrait être leur devise. C'est pourquoi, nous appellerons les PMI de ce dernier groupe les planificateurs « visionnaires ». Il semblerait que les groupes isolés puissent, à première vue, s'opposer deux à deux : d'une part, planificateurs-acteurs contre analystes et d'autre part, observateurs contre visionnaires. Cette tendance est confirmée par une analyse discriminante qui permet d'isoler les deux principaux axes différenciant chacun des quatre groupes de planificateurs : la propension à mener un diagnostic stratégique élaboré et la capacité à se projeter dans l'avenir. Au final, ces résultats sont synthétisés dans la figure 5 .

\section{CONCLUSION}

Près de 40 ans après l'introduction par les consultants de la planification à long terme dans les grandes firmes américaines, l'ambition de cet article était de s'interroger sur l'actualité de cette pratique, en France, dans les entreprises industrielles de petite et moyenne dimension. La planification d'entreprise, accompagnée de son lot d'outils, a en effet été l'objet d'une importante diffusion dans le milieu des affaires, aujourd'hui encore sous un vocable différent, et ce malgré le désintéressement relatif du milieu académique à l'égard de ce thème.

La littérature concernant les travaux de recherche sur la planification en PME suggère deux remarques: tout d'abord, il n'y a pas de consensus entre les auteurs pour mesurer la présence ou l'absence de la planification dans ces entreprises. Dans le même temps, les conclusions de recherches empiriques semblent converger vers le rejet de ce type de comportement, qualifié, lorsqu'il existe, d'informel, de sporadique et non structuré. Il nous a donc semblé indispensable, du fait d'une littérature sur la question à la fois riche et divergente, de nous positionner en termes conceptuels sur l'identification de la planification en entreprise. Il n'existe pas de consensus, en effet, sur la méthode à mettre en œuvre dans ce but, et chaque auteur, par ses choix, contribue un peu davantage à l'hétérogénéité du champ. C'est ainsi que nous avons été amenés à définir la planification comme un dispositif de gestion, c'est-à-dire une activité qui agit sur un objet, sans préjuger de ses nombreux domaines d'application potentiels. Ainsi, cinq dimensions caractérisent toute démarche de planification, dont l'identification en entreprise de trois d'entre elles (les sous-systèmes de finalisation, de programmation et d'action) nous paraît indispensable. Dès lors, nous avons envisagé une large enquête, de nature quantitative par questionnaires, auprès de responsables de PMI, afin d'évaluer leurs attitudes et pratiques face à la planification. Après avoir isolé 46 entreprises «planificatrices », nous avons recherché, à travers l'analyse rapide de quelques facteurs de contingence (tels que la taille, la propriété du capital ou bien le profil des dirigeants), quelques éléments de compréhension de leur comportement. Ces premiers résultats nous ont conduit, par la mise en œuvre d'une classification typologique, puis d'une analyse discriminante, à identifier quatre types de profils de PMI à l'égard de la planification: les « acteurs », les « analystes », les « observateurs » ainsi que les « visionnaires ».

Pour autant, comme nous l'indiquions dès l'introduction, le débat n'est pas clos. Parmi les prolongements possibles, et afin de répondre aux besoins des dirigeants de PME, il serait notamment souhaitable de 
tendre vers la recherche de démarches de planification à la fois souples et intégratives, en adéquation avec l'actualité des conditions d'exercice des tâches de direction. D'un point de vue plus conceptuel, il nous semblerait par ailleurs souhaitable de s'interroger sur la façon dont les démarches de planification en PME peu- vent s'inscrire dans un processus plus global de formation de la stratégie, dans la lignée des réflexions de Tannery et Metais (2001). À travers l'approfondissement de ces lignes de recherche, le monde de la recherche académique comme l'ensemble des praticiens auront sûrement à y gagner.

\section{BIBLIOGRAPHIE}

Ackoff R. L., Méthodes de planification dans l'entreprise, éd. d'Organisation, Paris, 1973. Ansoff H. I., Corporate Strategy: an Analytic Approach to Business Policy for Growth and Expansion, McGraw Hill, New York, 1965.

Bamberger I., «Situations et comportements stratégiques des petites et moyennes entreprises », Direction et Gestion, n 4, 1980, p. 21-30.

Bracker J. S. et Pearson J. N., "Planning and financial performance of small, mature firms", Strategic Management Journal, vol. 7, $\mathrm{n}^{\circ}$ 6, 1986, p. 503-522.

Calori R., Very P. et Arregle J. L., « Les PMI face à la planification stratégique », Revue française de gestion, $\mathrm{n}^{\circ}$ 112, janvier-février 1997, p. 11-23.

Desreumaux A., Stratégie, Dalloz, Paris 1993.

Drucker P. F., "Long Range Planning: Challenge to Management Science", Management Science, avril 1959, p. 238-249.

Goy H., Les réalités de la planification organisationnelle des petites et moyennes entreprises industrielles, Thèse de doctorat en sciences de gestion, Grenoble II, ESA, 16 novembre 2000.

Goy H., « De la nécessité de poursuivre la recherche sur la planification des PME », Revue Internationale PME, vol. 14, $\mathrm{n}^{\circ}$ 2, 2001.

Julien P. A. et Marchesnay M., La petite entreprise - Principes d'économie et de gestion, Éditions Vuibert, 1988.

Kargar J., "Strategic planning system characteristics and planning effectiveness in small mature firms", Mid-Atlantic Journal of Business, vol. 32, n 1, mars 1996, p. 19-34.

Marchesnay M., « La moyenne entreprise existe-t-elle? », Revue française de gestion, n 116 , novembre-décembre 1997, p. 85-94.

Martinet A. C., Stratégie, Paris, Vuibert, 1983.

Mintzberg H., Grandeur et décadence de la planification stratégique, éd. Dunod, Paris, 1994.

Montebello M. H., Saias M. et Greffeuille J., «Les PMI face à la planification stratégique », Papier de recherche, Stratégie et Structure, n 74-1, novembre 1974.

Ozbekhan H., "Toward a General Theory of Planning, Perspectives of Planning", (ed.) Jantsch E., Éditions de l'OCDE, 1969, p. 46-155. 
Paturel R., «Externalisation et entrepreneuriat », Histoire d'entreprendre et réalités de l'entrepreneuriat, coordonné par Verstrate T., Paris, éditions EMS Management et Société, 1999, p. 173-186.

Robinson R. B. et al., "The relationship between stage of development and small firm planning and performance", Journal of Small Business Management, vol. 22, n 2, avril 1984, p. 45-52.

Robinson R. B. et Pearce J. A., "The impact of formalized strategic planning on financial performance in small organizations", Strategic Management Journal, vol. 4, $\mathrm{n}^{\circ}$ 3, 1983, p. 197-207.

Robinson R. B. et Pearce J. A., "Research thrusts in small firm strategic planning", Academy of Management Review, vol. 9, $\mathrm{n}^{\circ}$ 1, 1984, p. 128-137.

Robinson R. B., Logan J. E. et Salem M. Y., "Strategic versus operational planning in small retail firms", American Journal of Small Business, vol. 10, n 3, 1986, p. 7-16.

Sexton D. L. et Van Auken P. M., "A longitudinal study of small business strategic planning”, Journal of Small Business Management, vol. 23, $\mathrm{n}^{\circ}$ 1, janvier 1985, p. 7-15.

Sexton R. N. et Dahle R. D., "Factors affecting long-range planning in the small business firm", Marquette Business Review, vol. 20, n 4, 1976, p. 158-165.

Shaw J. J., Shuman J. C. et Sussman G., “An empirical analysis of planning methods utilized by small New England business firms", The Northeast Journal of Business \& Economics, vol. 12, n² 2, printemps-été 1986, p. 54-67.

Shrader C. B., Mulford C. L. et Blackburn V. L., "Strategic and operational planning, uncertainty, and performance in small firms", Journal of Small Business Management, vol. 27, $\mathrm{n}^{\circ} 4$, octobre 1989, p. 45-60.

Shuman J. C., "Corporate planning in small companies, a survey", Long Range Planning, vol. 8, n 5, 1975, p. 81-90.

Steiner G. A., Strategic Planning : What Every Manager Must Know, New York, Free Press, 1979.

Tabatoni P. et Jarniou P., Les systèmes de gestion : politiques et structures, PUF, Paris, 1975. Tanguy H., « Planification stratégique: pour un usage rético-réthorique des modèles », Gérer et Comprendre, septembre 1992.

Tannery F. et Metais E., La création de la stratégie: éléments de repérage et propositions théoriques, Stratégies, actualité et futurs de la recherche, coordonné par Martinet A. C. et Thietart R. A., Paris, éditions Vuibert, 2001, p. 115-138.

Thietart R. A., La stratégie d'entreprise, McGraw Hill, Paris 1990.

Torrès O., « Pour une approche contingente de la spécificité de la PME », Revue internationale PME, vol. 10, $\mathrm{n}^{\circ} 2$, 1997, p. 9-43.

Vancil R. F., Strategy Formulation in Complex Organizations, Strategic Planning Systems, Lorange P. and Vancil R. F. (ed.), Englewood Cliffs, Prentice Hall, 1977, p. 4-22.

Waalewijn P. et Segaar P., "Strategic management: the key to profitability in small companies”, Long Range Planning, vol. 26, n² 2, 1993, p. 24-30. 